\title{
Voltage regulation in LV grids by coordinated volt-var control strategies
}

\author{
Miguel JUAMPEREZ, Guangya YANG ( $\square)$, \\ Søren Bækhøj KJER
}

Keywords Voltage regulation, Reactive power optimization, Genetic algorithm

\section{Introduction}

The governmental goals of greenhouse gas (GHG) emission reduction along with the cost reduction in technology, are the main driving factors for the rapid penetration of renewable energy source (RES) [1]. The factors supporting the diffusion of RES in the system include the liberalization of energy markets, the increasing retirement of fossil-fuelled and nuclear energies due to environmental awareness and the large potential of renewable energy technologies, such as wind, solar or hydropower working in a local scale.

As a reliable resource, the solar photovoltaic (PV) contributes to a successful integration of renewable energy generation by the new sustainable energy scheme. However, the distributed grid-connected PV poses some similar challenges: the mismatch between the production and the demand due to the stochastic generation of PV, the discontinuity and bidirectional power flow which can affect the loading of infrastructure and the operation of protection systems, the voltage rise issues at the point of connection and neighbor buses amplified by the small $\mathrm{X} / \mathrm{R}$ ratios at low voltage (LV) feeders and the potential overloading of network equipments [2].

For this reason emerging smart grid solutions, such as load management [3], energy storage [4] or virtual power plants [5], are gradually adopted in medium and low voltage grids to mitigate the operational problems of solar power plants based on the state-of-the-art of the information communication technologies (ICTs), under the network operator surveillance [6].

The voltage rise is one of the major issues experienced in LV grids with high share of PVs [7]. The voltage rise 
due to active power injection and small $\mathrm{X} / \mathrm{R}$ ratios in $\mathrm{LV}$ feeders restrict the amount of power to be installed in a hosting network. As a result, customers located at the end of the line will suffer from undesired overvoltage, as shown in Fig. 1, which may result inverters stopping operation, as defined by some grid codes, e.g., VDE AR-N 4105 [8]. Hence, the system operators acquire more services from the solar inverters connected to the grid in order to increase the penetration level and facilitate the operation.

The main strategies to increase the PV integration in LV networks mentioned in $[9,10]$ are:

- Network feeders upgrade despite the effects in the protection system and high cost [11].

- Reactive power absorption to decrease the voltage at the point of connection [12].

- Active power curtailment with a common beneficial level for producers and consumers [13].

- Adjust the output voltage of the distribution transformer by maneuvering the tap changer [14].

- Load management to match production and consumption one day in economic manner [15].

- Utilization of storage to approach self-supply [16].

The study of voltage control in distribution systems also concerns inverter manufactures as one of the critical issues in PV integration, and solar inverters are required to participate actively in grid security under the command of the system operator. Accurate system models are necessary to reach an optimal design of the inverter controller for that purpose. This paper describes a testing platform developed to optimize the voltage and the power losses in a sub-urban LV network with different levels of PV penetration. Coordinated control strategies contribute positively to voltage regulation as described in the following sections.

\section{Problem}

Traditionally, the voltage in transmission and distribution networks has been regulated through dispatch of active

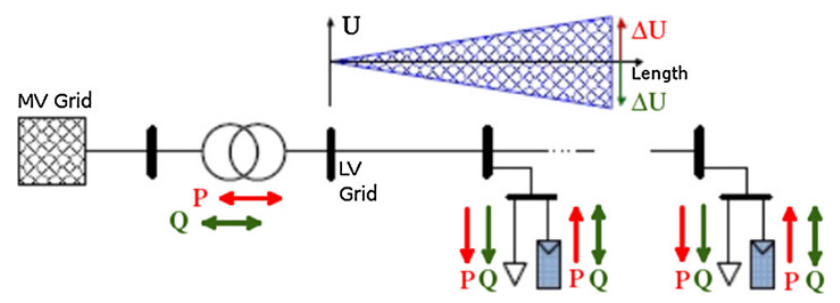

Fig. 1 Bidirectional power flow and voltage fluctuations in LV networks with PV integration [17] and reactive powers from stand-alone centralized plants. Generators, shunt capacitors, STATCOMs, and SVCs collaborate to maintain the voltage within limits under every load condition. Theoretically, the voltage control can be performed in a similar way in distribution networks based on a management system controller with costly communication equipment. The increasing distributed and local power injection in medium voltage (MV) and LV grids alters the network operation and intensify the concerns regarding the bidirectional power flow effect in voltage control.

The substantial resistive value of distribution lines forces the transformers to make their contribution in voltage regulation. At primary substations, on-load tap-changer (OLTC) transformer maneuvers the voltage downstream according to the sensor and relay commands. Unfortunately, the reverse current flow restricts its reliability. New generations of electronic switches in conjunction with vacuum chamber solutions [18], are under research with the premise of significant enhancement of consumer's voltage profile. Reducing the voltage bandwidth at the distribution transformer, would allow higher PV penetration level with the risk of reaching under voltages in the feeders connected to the transformer. Furthermore, this action would increase the tap change operation frequency and hence, drive some customers to experience power supply difficulties. As a consequence, a second voltage control strategy is necessary.

The high versatility of solar inverters in reactive power management makes them desirable to mitigate the overvoltage phenomena. Ref. [19] provided four local reactive power control concepts: fixed power factor, constant reactive power, local reactive power control dependent on the voltage $\mathrm{Q}(\mathrm{U})$, and power factor control dependent on the power injection $\cos \varphi(\mathrm{P})$. The last two techniques are of interest for this study as they claim to mitigate overvoltage situations considering the inverter working conditions unlike the fixed parameter ones. The downsides to the approach include the overrating of inverters and the possible increment of losses as a result of reactive current circulation.

In brief, the most common requirements for coordinated volt-var power control are as follows:

- Keep bus voltages within limits;

- Minimize the active power losses and the number of tap change operations;

- Manage the reactive power source;

- Regulate the transformer and the feeders loading;

- Control the power factor.

This paper proposes a coordinated solution for matching the reactive power capabilities of solar inverters and the 


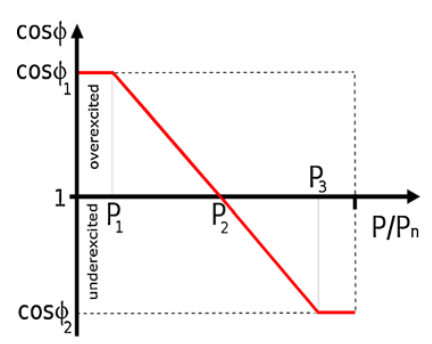

(a)

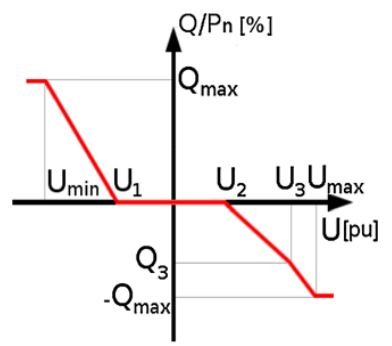

(b)
Fig. 2 Reactive power curves a $\cos \varphi(\mathrm{P})$ curve, b $\mathrm{Q}(\mathrm{U})$ curve

grid voltage regulation of the LV distribution transformer, to mitigate the voltage rise issue in a $\mathrm{LV}$ network.

The weakness of distribution networks (low X/R ratio) forces the absorption of large amount of reactive power by the solar inverters to significantly mitigate the voltage rise. Due to the increase of the distributed generation in the power systems, its impact on the network becomes significant. Therefore, standards are extended to comply with the new electrical scenario where the effects of power fluctuations on neighbor buses voltage cannot be neglected. Ref. [20] specified the voltage range criterion for every component in the $0.4 \mathrm{kV}$ and $10 \mathrm{kV}$ grids as:

$-10 \% \leq \Delta V \leq 10 \%$.

Higher deviations are considered as under/over voltages.

\section{Reactive power capabilities of PV}

The reactive power capabilities of solar inverters can be used to maintain the voltage level within the limits. The effectiveness of the active power control on voltage support in LV systems increases with the distance or resistance to the distribution transformer whereas the reactive power does mostly to the inductance value of the transformer. Thus, inverters located at the end of the feeder would work on low PF values, potentially increase the losses of the grid.

From the above volt-var strategies recommended, the fixed methods are not examined since as a result of the fluctuating power production of PV panels a variable displacement factor is preferable. Thereby, the power factor dependent on injected active power $(\cos \varphi(\mathrm{P}))$ and reactive power as a function of the voltage at the terminal $(\mathrm{Q}(\mathrm{U}))$, are implemented as standard reactive power control methods [21, 22]. The option of $\cos \varphi(\mathrm{U})$ control is not considered as the current used inverter which does not have the function at the time of test. The first strategy adjusts the reactive power flow back to the grid based on the level of the inverter active power output, thus regulating the voltage. According to Fig. 2, at low production levels inverters may be required to inject reactive power by the distribution system operator (DSO). Nevertheless, the assumption of the voltage rise as a consequence of active power increment regardless the load variation may lead to reactive power absorption under normal voltage conditions, which is one of the drawbacks of this concept. Eq. (2) expresses the $\cos \varphi(\mathrm{P})$ curve shown in Fig. 2(a).

$\cos \varphi(\mathrm{P})= \begin{cases}\cos \varphi_{1}, & P \leq P_{1} \\ \cos \varphi_{1}+\left(1-\cos \varphi_{1}\right)\left(\frac{P_{1}-P}{P_{1}-P_{2}}\right), & P_{1}<P \leq P_{2} \\ -1+\left(1+\cos \varphi_{2}\right)\left(\frac{P_{2}-P}{P_{2}-P_{3}}\right), & P_{2}<P \leq P_{3} \\ \cos \varphi_{2}, & P>P_{3}\end{cases}$ $\varphi_{1}>0, \varphi_{2}<0$.

The local voltage information monitored in each inverter is used by $\mathrm{Q}(\mathrm{U})$ method to respond accordingly and hence, reduce reactive power absorption losses. Due to the line impedance awareness of this procedure, an overuse of inverter reactive power capabilities is minimized. The limited reactive power capability of local inverters along with the possible misuse of the available power resources near the transformer, leads to the use of a coordinated strategy. A coordinated solution with OLTC transformers is suggested.

$$
Q(\mathrm{U})= \begin{cases}Q_{\max }, & U<U_{\min } \\ \frac{U-U_{1}}{U_{\min }-U_{1}} Q_{\max }, & U_{\min } \leq U<U_{1} \\ 0, & U_{1} \leq U \leq U_{2} \\ \frac{U-U_{2}}{U_{3}-U_{2}} Q_{3}, & U_{2}<U \leq U_{3} \\ \frac{U_{\max }-U}{U_{\max }-U_{3}} Q_{3}-\frac{U-U_{3}}{U_{\max }-U_{3}} Q_{\max }, & U_{3}<U \leq U_{\max } \\ -Q_{\max }, & U>U_{\max }\end{cases}
$$

The $\mathrm{Q}(\mathrm{U})$ characteristic curve implemented is shown in Fig. 2(b). It is worth noting that two droop ratios are available when the voltage is higher than the normal range. Besides, achieving better voltage control functions can differentiate the voltage responses of the inverters near LV transformers from the rest along the feeder, so the reactive power contributions from all inverters along the feeder can be more equally distributed.

From the available coordinated control methods for LV networks with increasing diffusion of PV power, a voltage regulation performed by an OLTC $\mathrm{MV} / \mathrm{LV}$ transformer (central) plus a reactive power control in the solar inverter (local), is proposed. A graphical representation is shown in Fig. 3.

The inverter controller model in Fig. 4 based on [23], includes open loop reactive power controls and network voltage regulation. The active power to be delivered into the grid $\left(P_{g e n}\right)$ is ordered by a solar power profile userdefined $\left(P_{\text {ord }}\right)$, and a standard power curtailment is 


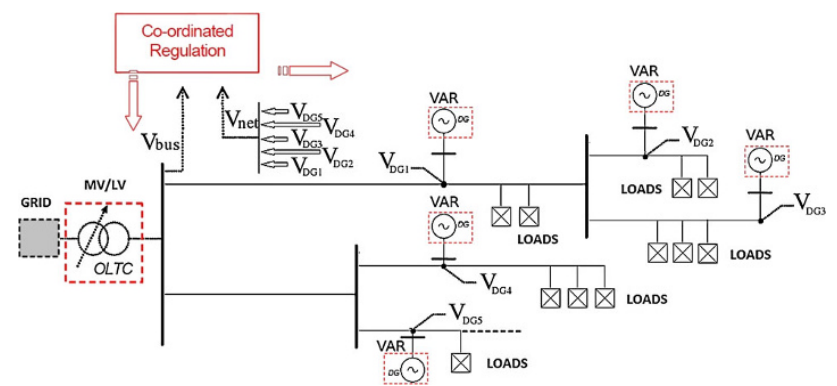

Fig. 3 Converter var control model for distributed solar inverters

available to comply with the DNO requirements. On the contrary, the reactive power control generated $\left(Q_{\text {gen }}\right)$ is based on a supervisory var power output $\left(Q_{\text {ord }}\right)$ which may come from either of the two characteristic var curves described above.

The electrical model takes the active and reactive power commands as inputs to compute the active and reactive current commands using the terminal voltage $\left(V_{\text {term }}\right)$. Compared the commanded reactive power current with the current reactive power current, the difference is integrated with an adjustable PI controller to meet the desired reactive current command. Thus, a rise at terminal voltage will result in an increment of reactive power command according to the selected var control method. Then, a three phase controlled-current source injects the ordered current into the grid.

As a secondary voltage control, a network voltage regulation strategy is implemented at the distribution transformer level. OLTC transformers are capable of stepping up and down the voltage downstream without interrupting the power supply or causing disturbances to the consumers connected at the end of the lines. All bus voltages with PV connection plus the low voltage terminal of the transformer are used as inputs to obtain the weighted average voltage magnitude of the network. This value is compared with a pre-defined voltage boundary to adjust the tap changer position. The importance of setting a proper voltage boundary for the tap changer to actuate makes its design harder. As drawbacks, residents closest to the transformer may suffer undesirable undervoltage values and the OLTC transformer technology is still under research and test for LV networks.

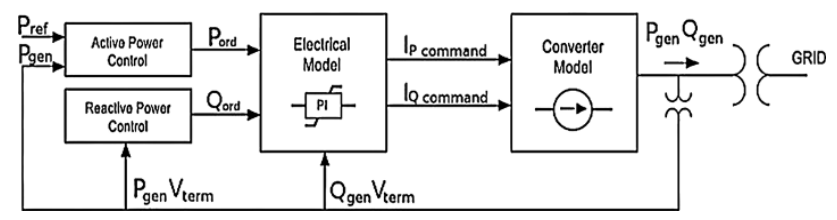

Fig. 4 Schematic diagram of coordinated volt-var regulation [24]

\section{Voltage control optimization}

The effects of the increasing utilization of DGs on the grid are directly related to the location, size and type of generation as well as the system topology. The target of finding the optimal PV penetration in a LV network in the sense of voltage and power losses values can be formulated as a non-linear multi-objective optimization problem. In short, the reactive power sources are allocated so that the active power losses of the grid are minimized and the voltage security margin can be maximized. The presence of multiple local maxima or minima in the objective function drove the scientists to develop evolutionary algorithms as the genetic algorithm (GA) at the expense of conventional methods, such as gradient-based algorithm. GA is inspired by natural selection mechanisms where the individuals with the best fitness values from a random initial population are selected to enhance the generation. In this case, the proposed algorithm identifies the optimal bus voltage magnitudes, transformer tap settings and reactive power outputs. The optimization problem includes:

\subsection{Optimization variables}

Weighting factors $w_{i}(i=1: 4)$ of voltage measures along the feeder to formulate the OLTC voltage reference. The feeder is divided into 4 areas and the average voltages in each area are used [25]. Reactive power curve parameters $\cos \varphi_{1}, \cos \varphi_{2}, U_{1}, U_{2}, U_{3}, Q_{3}$ are shown in Fig. 2. $P_{\mathrm{i}}$ presents $i$-th inverter active power generation level which is used to determine the best penetration level of PV. The limit of $0.6 \mathrm{pu}$, which represent $60 \%$ penetration of $\mathrm{PV}$ $(216 \mathrm{~kW})$ set by the transformer and cable capabilities.

\subsection{Static parameters}

$V_{\text {Lmax }}, V_{\text {Lmin }} \quad$ Bus voltage limits

$S_{\text {imax }} \quad$ Flow limit of the i-th feeder section

$Q_{\mathrm{Gmax}}, Q_{\mathrm{Gmin}} \quad$ Inverter reactive power limits

It is worth noting that the bus voltages further from the transformer station are worse than the ones near the transformer, as shown in Fig. 1. Therefore, it is necessary to take this into consideration when defining the voltage reference for the OLTC. In this work, the buses are organized into zones and parameters' weighting are given to the buses further from the transformer. Certainly, more options can be proposed.

Regarding the $\cos \varphi(\mathrm{P})$ curve, an introduction of a deadband bracketing $0.5 \mathrm{pu}$ is initially tested without benefits in voltage or losses over the final selected solution. Therefore, only $\cos \varphi_{1}$ and $\cos \varphi_{2}$, are deployed in the 
variable list. This is the main reason to choose few points to define the reactive power control curves.

Having two main objective functions, voltage profile and power loss, multi-objective genetic algorithm (MOGA) is the best option for highly constrained optimization problems. MOGA is used in this case for its efficiency and simplicity methodology with the evolutionary algorithms. GA optimizes a given fitness function whose objectives are the loss minimization and the voltage stability margin maximization. The minimization of losses will be followed by an efficiency rise and hence, by a cost reduction. The static parameters are satisfied by adding penalty terms in the objective function. The overall fitness function can be written as

$$
\begin{aligned}
f= & f_{1}+f_{2}+\lambda_{1} \sum_{i=1}^{N_{T}} \Delta V_{i_{L}}^{2}+\lambda_{2} \sum_{i=1}^{N_{I}} \Delta Q_{i_{G}}^{2} \\
& +\lambda_{3} \sum_{i=1}^{N_{L}}\left(S_{i}-S_{i}^{\max }\right)^{2},
\end{aligned}
$$

where $f_{1}$ is the active power loss on the transformer and feeder, $f_{2}=\sum\left|V_{i}-V_{\text {ref }}\right|\left(V_{\text {ref }}=1\right)$ is the voltage deviation at every bus with PV installation, and $\lambda_{1}, \lambda_{2}, \lambda_{3}$ are the penalty factors.

The penalty functions are defined as:

$$
\begin{aligned}
& \Delta V_{i_{L}}=\left\{\begin{array}{c}
V_{L}^{\min }-V_{i_{L}}, V_{i_{L}}<V_{L}^{\min } \\
V_{i_{L}}-V_{L}^{\max }, V_{i_{L}}>V_{L}^{\max },
\end{array} \quad i=N_{T}\right. \\
& \Delta Q_{i_{G}}=\left\{\begin{array}{c}
Q_{G}^{\min }-Q_{i_{G}}, Q_{i_{G}}<Q_{G}^{\min } \\
Q_{i_{G}}-Q_{L}^{\max }, Q_{i_{G}}>Q_{G}^{\max }
\end{array}, \quad i=N_{I}\right.
\end{aligned}
$$

$S_{i} \leq S_{i \max }, i=N_{L}$,

where $N_{I}$ is the number of buses, $N_{T}$ is the number of buses including transformer bus, and $N_{L}$ is the number of lines.

The power flow constraints are handled in time series simulation whereas the inequalities define the system operating boundaries, namely, voltage constraints, reactive power capabilities and cable loading limits. The $f_{1}$ in the objective function uses the aggregated value over the simulated period which shows the energy loss. The voltage is taken from the worse value during the simulation.

It is worth mentioning that as the problem is considered as a planning problem, where the control parameters are tuned for a period of time, therefore the computational time of GA is not a central issue considered here.

\section{Grid model and data description}

The representative LV network configuration of Denmark power systems, as shown in Fig. 5, is located in Bornholm Island. The grid is composed of one LV/MV distribution transformer and its downstream connections.

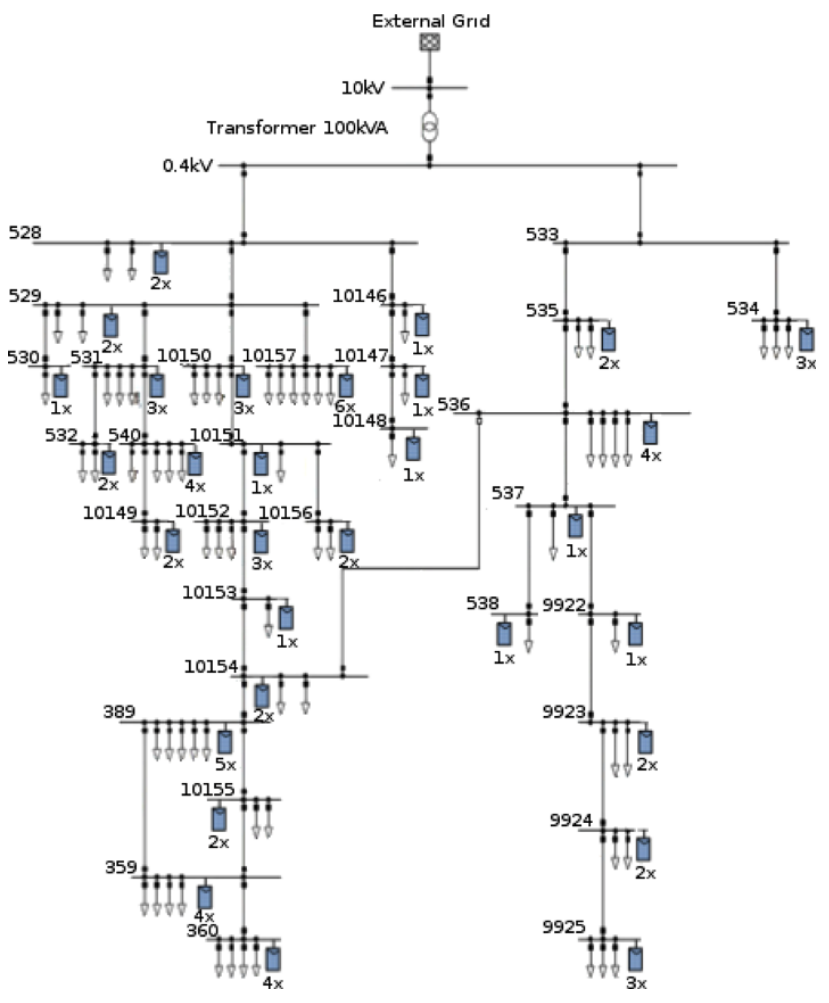

Fig. 5 Bornholm LV network layout under study

The grid supplies electricity to 71 consumers of a residential area through a $100 \mathrm{kVA} 10 / 0.4 \mathrm{kV}$ Dyn5 power transformer and two main radial feeders distributing at 0.4 $\mathrm{kV}$. The backup interconnection may facilitate the integration of local generation, but it is disconnected to study the worst case voltage rise scenario. More detailed description of the network can be found in [7].

According to long-term estimations and profitable rooftop PV power investment, the solar installation is defined at maximum $5 \mathrm{kVA}$ per consumer in residential areas [7]. By this definition, a uniform energy distribution along the feeders may be achieved. Eq. (6) shows the definition of PV penetration percentage $L_{P V}$ by using total installed maximum PV inverter capacity $S_{P V}$ feeder, the number of consumers $n_{\text {loads }}$ and maximum inverter capacity $S_{r}$ of each consumer.

$S_{P V \text { feeder }}=L_{P V} \cdot n_{\text {loads }} \cdot S_{r} / 100[\mathrm{kVA}]$.

Power generation measurements in 15 min average of a $5 \mathrm{kVA}$ installation for a week are used as PV profile for simulation, as shown in Fig. 6. Consumption data from four representative Danish residences in Fig. 7, are selected [26]. The reason of the consumption level difference is the use of electric heating and the type of dwelling, house or apartment. For simulation purposes, consumption data are transformed into 15 min average. Identical production and consumption profiles are shared 


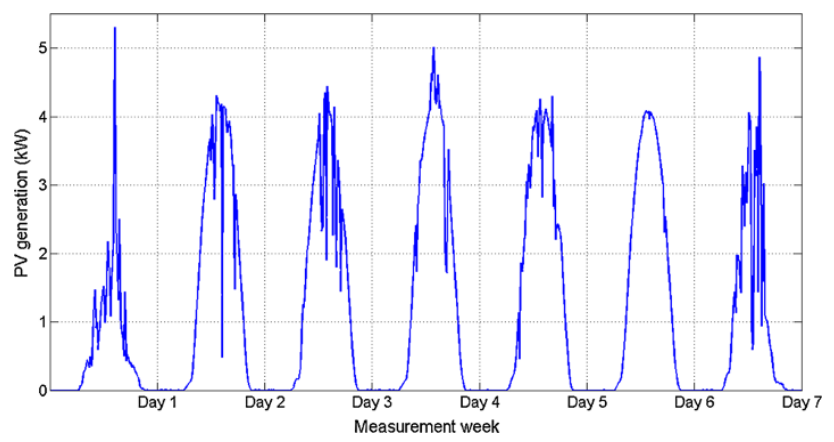

Fig. 6 PV output profile of a week from a 5 kWp system (Aug. 2013)

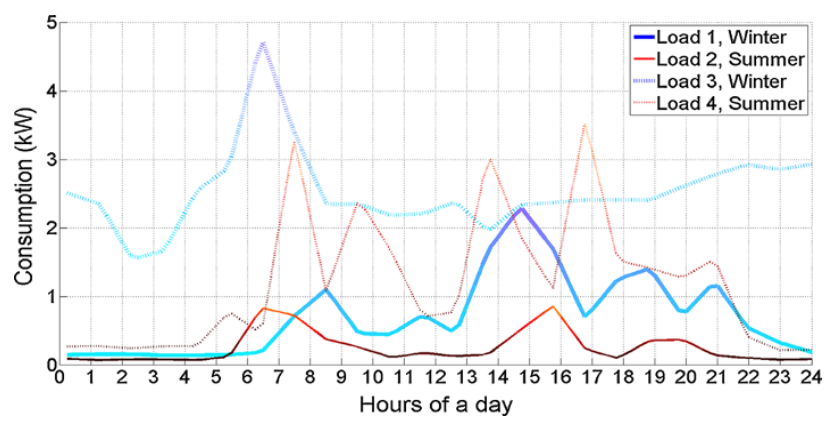

Fig. 7 Danish consumption profiles utilized from 4 households

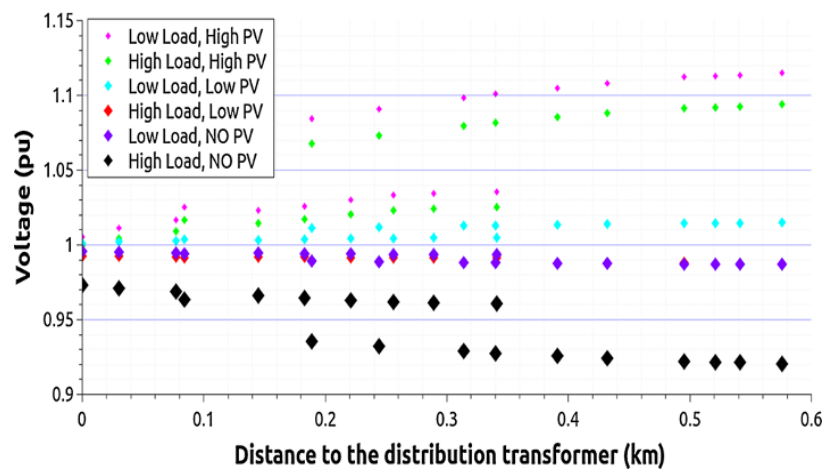

Fig. 8 Voltage profile comparison with several loads and PV conditions

by all buses in each steady-state voltage simulation case. Though the used load profiles include both in summer and winter, PV production data from summer are only used to stimulate the worst case of the voltage rise.

Fig. 8 shows the voltage magnitudes obtained in the network under study (Fig. 5) for different loads and PV generation conditions, where:

- $\quad$ Low load $=$ Load 2 (Summer)

- $\quad$ High load $=$ Load 4 (Summer)

- Low PV $=10 \% \mathrm{PV}$ penetration
- High PV $=60 \%$ PV penetration

It can observe the correlation between the PV penetration and the load levels; the lower the load the higher the voltage values for the same PV power injected. The overvoltage boundary starts to be exceeded at penetration levels around 60\% (213 kVA). On the other hand, solar power contributes to avoiding costumers at the end of the feeders to experience critical low voltage values in case of large demand conditions.

\section{Results}

Table 1, Figs. 9-10 collect the best fitness function and parameter values for both var regulation methods (according to Fig. 2) for each load case of optimal PV generation obtained. $\mathrm{U}_{\min }, \mathrm{U}_{\max }$ are voltage boundaries $5 \% /+5 \%$ while $\mathrm{P}_{1}, \mathrm{P}_{2}, \mathrm{P}_{3}$ are defined as $0.15,0.5$ and 0.85 $\mathrm{pu}$, respectively, due to the similar values of every case. In summary, PV penetrations from $3 \%$ to $20 \%$ (10.6-71 kVA) are obtained depended on the demand level of each consumer. And the higher the consumption, the higher amount of solar power is accommodated within the network loading restrictions and minimum losses. From the point of view of the transformer capability, a minimum installation of $100 \mathrm{kVA}$ should be possible. With regard to parameter values of the control strategies, it can observe a clear homogeneity between simulation cases. For comparison, voltage, losses and transformer loading status magnitudes are presented in Figs. 11-13, respectively.

\subsection{Voltage}

The PV power profile given in Fig. 6 creates critical voltage levels $(>1.1 \mathrm{pu}$, yellow line) at the end of the feeder when the amount of active power injected into the network exceeds $60 \%$ for all loading cases. Voltage magnitudes along the feeders shown in Fig. 11 can compare results of applying different reactive power control curves with/without OLTC voltage control with a representative loading case (Load 4), at diverse PV penetration levels for the most significant hour of the profile (1-2 pm). Load 4 profile is considered to be the most suitable for sharing the same measurement time (summer) with the PV production. The tap changer voltage boundary, based on a weighted average network value, is set at $\pm 5 \%$ of the reference value $(1 \mathrm{pu})$. The grayish area symbolizes the bus voltage above $1.05 \mathrm{pu}$ which may lead to a tap change in the transformer if the average exceeds this boundary. Despite that all inverters under $\cos \varphi(\mathrm{P})$ configuration contribute equally to the grid voltage support, the overvoltage limit is reached 
Table 1 Optimized Pareto and parameter values

\begin{tabular}{|c|c|c|c|c|c|c|c|c|c|c|c|c|}
\hline \multirow[t]{2}{*}{ Parameter } & \multicolumn{3}{|c|}{ Load 1} & \multicolumn{3}{|c|}{ Load 2} & \multicolumn{3}{|l|}{ Load 3} & \multicolumn{3}{|l|}{ Load 4} \\
\hline & $\begin{array}{l}\text { No } \\
\text { PV }\end{array}$ & $\cos \varphi(\mathrm{P})$ & $\mathrm{Q}(\mathrm{U})$ & $\begin{array}{l}\text { No } \\
\text { PV }\end{array}$ & $\cos \varphi(\mathrm{P})$ & $\mathrm{Q}(\mathrm{U})$ & No PV & $\cos \varphi(\mathrm{P})$ & $\mathrm{Q}(\mathrm{U})$ & No PV & $\cos \varphi(\mathrm{P})$ & $\mathrm{Q}(\mathrm{U})$ \\
\hline$f_{1}(\mathrm{~kW})$ & 8.503 & 7.692 & 8 & 6.794 & 6.5635 & 6.5905 & 30.229 & 29.281 & 28.381 & 12.953 & 11.146 & 11.531 \\
\hline$f_{2}(\mathrm{pu})$ & 0.466 & 0.1526 & 0.1654 & 0.169 & 0.0702 & 0.0711 & 1.381 & 1.492 & 0.499 & 0.787 & 0.25107 & 0.2591 \\
\hline $\begin{array}{l}\text { Optimal PV } \\
\text { penetration }(\%)\end{array}$ & & 9.6 & 9.55 & & 2.9 & 2.88 & & 18.55 & 18.8 & & 14.39 & 15.02 \\
\hline $\cos \varphi_{1}$ & & 0.9519 & & & 0.9509 & & & 0.961 & & & 0.9483 & \\
\hline $\cos \varphi_{2}$ & & -0.934 & & & -0.941 & & & -0.941 & & & -0.944 & \\
\hline$U_{1}$ & & & 0.957 & & & 0.961 & & & 0.979 & & & 0.979 \\
\hline$U_{2}$ & & & 1.018 & & & 1.021 & & & 1.020 & & & 1.022 \\
\hline$U_{3}$ & & & 1.047 & & & 1.045 & & & 1.045 & & & 1.048 \\
\hline$Q_{3}$ & & & -0.28 & & & -0.275 & & & -0.28 & & & -0.32 \\
\hline
\end{tabular}

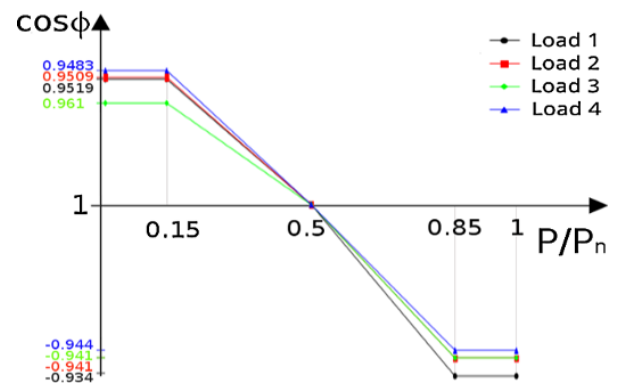

Fig. $9 \cos \varphi(\mathrm{P})$ curve parameters for different loading cases

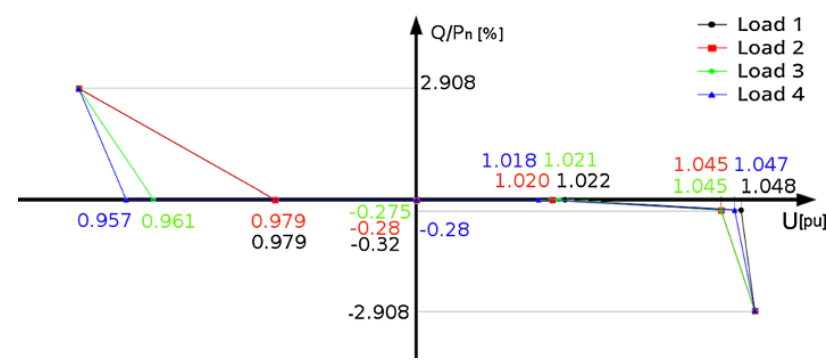

Fig. $10 \mathrm{Q}(\mathrm{U})$ curve parameters for different loading cases

around $60 \%$ of solar integration (green line). However, with coordination with OLTC, the voltage level drops about $0.03 \mathrm{pu}$ within the limits. On the contrary, when $\mathrm{Q}(\mathrm{U})$ method is activated, bus voltages remain closer to the reference throughout the line length, especially with large PV power distributed.

\subsection{Power losses}

The disparity in losses grows as the distributed power installation increases. According to Fig. 12, Q(U) strategy achieves lower grid power losses generally compared with

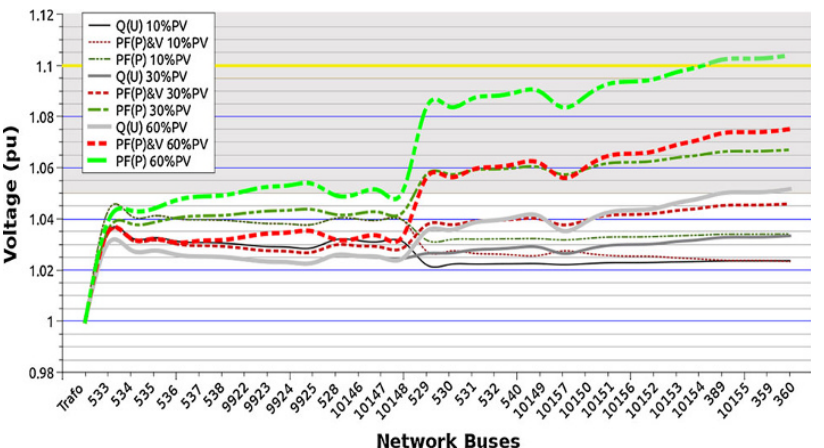

Fig. 11 Node voltage comparison between reactive power control curves and effect of the coordinated voltage control

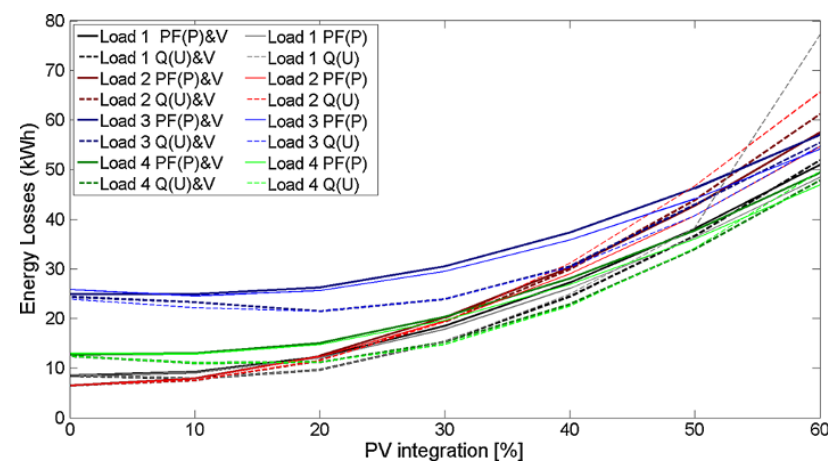

Fig. 12 Network power loss comparison between reactive power control curves and the effect of the coordinated voltage control

$\cos \varphi(\mathrm{P})$. However, at high solar power integration levels, the reactive absorption rises due to the voltage boundary proximity of the inverters located at the end of the line which causes greater power losses. In fact, a large consumption portfolio involves high current flow and therefore, high power losses. The benefits of an OLTC 


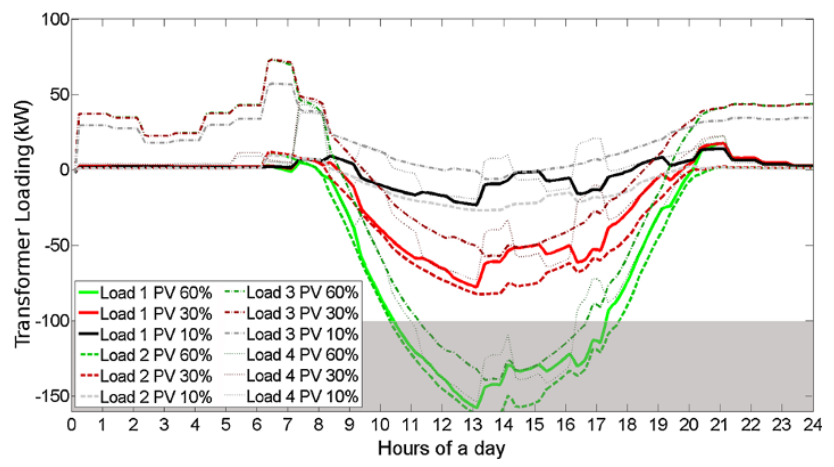

Fig. 13 Transformer loading levels under different consumptions and PV penetration conditions

transformer support are mainly neglected here. The main driver of losses growth with the power is the cable losses, as the transformer losses increase slowly.

\subsection{Feeder loading}

Feeder lifetime strongly depends on the loading conditions i.e., in network voltage variations. The cables are designed to withstand up to $250 \mathrm{~A}$ which approximately corresponds to around $50 \%$ of PV power penetration. Step down the voltage with the transformer contributes to slightly increasing the load flow through the feeders which may affect the lifetime of the cables and the transformer itself. Fig. 13 depicts the transformer loading level for diverse load and PV power conditions where under the worst-case load scenario, $60 \%$ PV penetration turns the distribution transformer to overloading conditions (shaded area) during peak production hours. In contrast, the transformer loading always remains functioning under its the nominal loading value with 30\% PV integration. Therefore, considering the transformer rating as a limiting parameter, the highest hosting capacity of the network is defined around $40 \%$ corresponding to $140 \mathrm{kVA}$. The same result is achieved by $[8,12]$. The production and demand profiles mismatch limits the network hosting capacity, which still can be enhanced by implementing a load management.

\section{Hardware implementation}

A practical setup is performed in the laboratory with a single transformer less Danfoss inverter of $10 \mathrm{kVA}$ rated power connected to $6 \mathrm{~kW}$ rooftop PV plant. A user data protocol (UDP) is used to transfer the data at real-time from the inverter to the user and back, through Ethernet communication. Information share between hosts on an internet protocol (IP) network without prior communications makes UDP useful for solar power plants with dozens

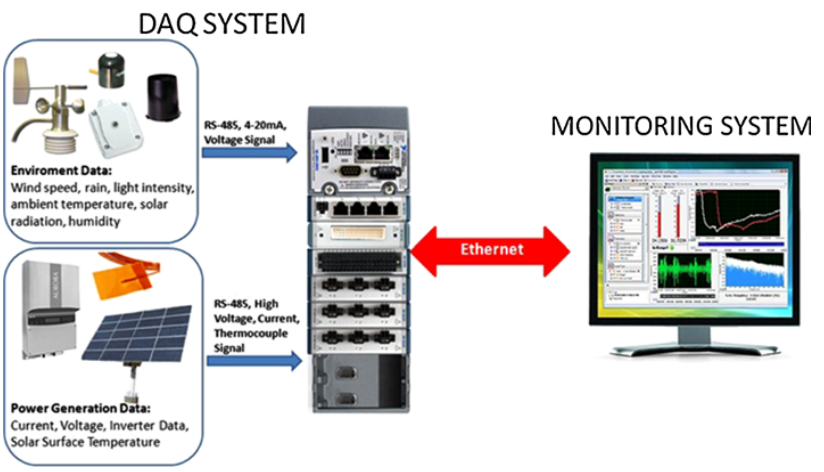

Fig. 14 Communication platform for real-time reactive power parameter regulation with TLX Danfoss inverter [27]

of inverters interconnected. Data measurements are made by a flexible embedded control and acquisition system with a solid and reconfigurable field-programmable gate array (FPGA) chassis named CompactRIO (cRIO). A communication platform is developed to control the var parameters of the inverter, as shown in Fig. 14, to minimize the losses and keep the terminal voltage within limits.

The modularity of cRIO chassis allows its implementation on a wide range of applications. For this specific case, two modules are selected, NI 9225 and NI 9227, to measure phase voltages and currents, respectively. Both modules are directly connected to the $\mathrm{AC}$ output of the inverter. All measurements are read and sent, according to a FPGA target, to the host computer which processes the data, continuously compares the voltage values to a reference in order to set the most suitable reactive power strategies and writes them in the inverter control board.

A block diagram describing the aforementioned functions is shown in Fig. 15. The scheme is divided into 8 sections, each of which stands for a function:

1) FPGA target definition. Input variables and constants, such as nominal voltage and frequency, wiring settings, sample frequency, are defined.

2) Specifying the basic configuration of the system and start FPGA measurement, voltage and current storage in arrays.

3) Reading data from the FPGA module FIFO of the realtime target for real-time systems.

4) Building voltage and current waveforms from input data, sampling rate of the input data, and the real-time controller time.

5) Building active and reactive power waveforms from voltage and current data.

6) Defining reactive power control mode and parameters based on voltage level and DSO requirements.

7) Calculating the one-cycle fundamental power values and RMS values of voltage and current. 


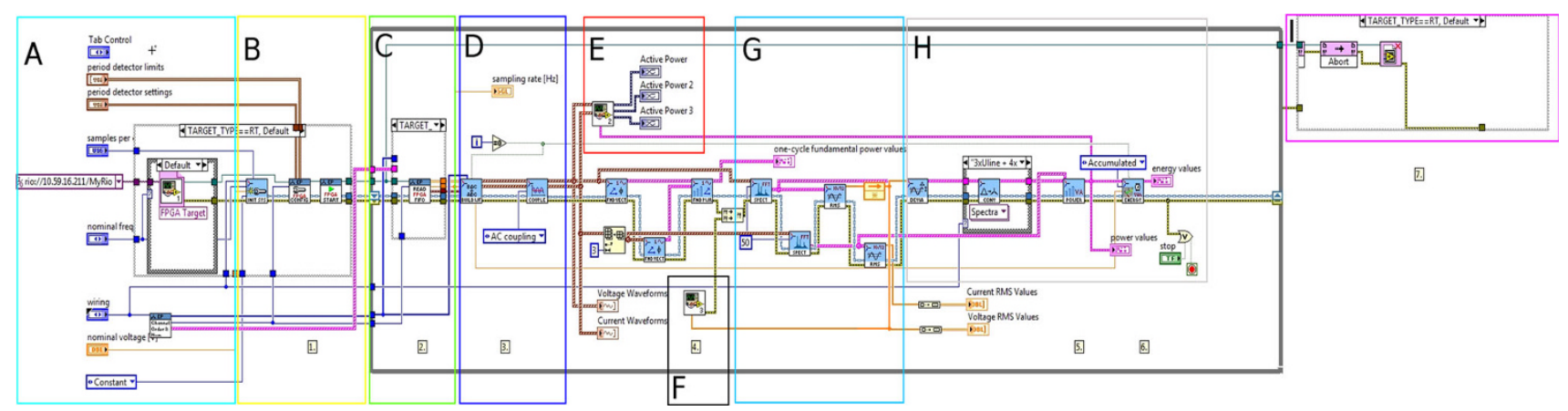

Fig. 15 Implementation diagram of cRio control circuit

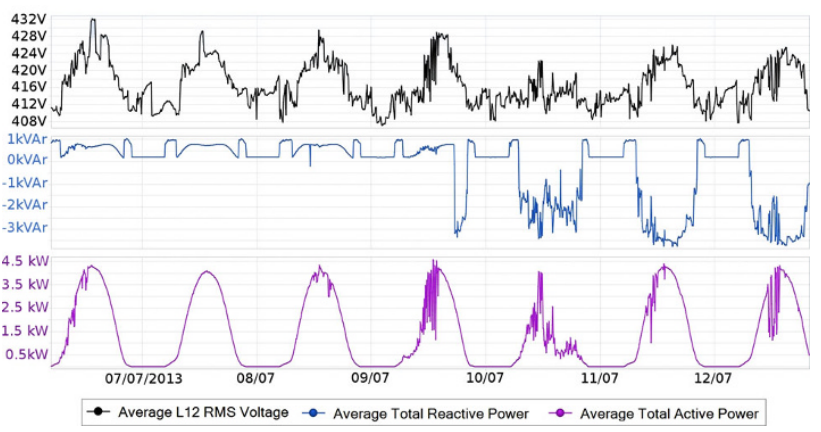

Fig. 16 Voltage comparison with $\mathrm{Q}(\mathrm{U})$ mode set (without OLTC)

8) Calculating power from the voltage and current signals and energy values from the power values.

With the aim of magnifying the voltage variability at the output terminals of the inverter under study, the impedance between the inverter AC output and the grid is enlarged by adding $100 \mathrm{~m}$ long and $10 \mathrm{~mm}^{2}$ cross-sectional area cable. Voltage magnitudes in Fig. 16 show the measurements obtained with a power quality analyzer, Elspec G4400 Series BlackBox hardware [28]. A voltage drop is observed when the inverter absorbs reactive power at the connection point.

\section{Conclusion}

A volt-var coordinated strategy is proposed to reach the optimal PV penetration level in a specific LV network by means of power losses and voltage deviations minimization. According to the simulations aforementioned, this value varies from $5 \%$ to $40 \%$ (17.8-142 kVA) of PV power distributed equally throughout the grid without having to reinforce the lines. At the same time, power flow reduction and energy savings are achieved by local power production.

Both reactive power management methods, $\cos \varphi(\mathrm{P})$ and $\mathrm{Q}(\mathrm{U})$, have beneficial effects on network voltage security which can be amplified by the support of an OLTC distribution transformer. Indeed, modify the voltage sensitivity of the farthest inverters to the transformer by setting a deadband around the reference value in $\mathrm{Q}(\mathrm{U})$ strategy, decreases the reactive power absorption and the losses. Increasing the size of the transformer would enlarge its lifetime, but it will not solve the network voltage problem. The hosting capacity can be enhanced by maneuvering the OLTC transformer.

When the reactive power capabilities are insufficient to regulate the voltage, a coordinated solution with an OLTC distribution transformer or an active power curtailment, as a last option, may be the solution. However, feeders with unbalanced solar power distribution supplied by the same transformer might present additional voltage issues unsolvable with this method. It is believed that in the upcoming years, communication systems will be completely spread as an affordable solution for distribution network operation. Ideally, a central communication network could command all inverters installed in a solar plant to the most appropriate volt-var strategy at each time. A cRIO hardware device capability of handling real-time measurements and setting control parameters in a solar inverter through a secure Ethernet communication network has been positively tested in the laboratory. Its implementation in large scale can save time and cost.

Acknowledgement The authors would like to thank distribution system operators Østkraft Net A/S and EnergiMidt A/S for their contributions to this paper. This work was supported in part by PVNET.dk project sponsored by Energinet.dk under the Electrical Energy Research Program (ForskEL, grant number 10698).

Open Access This article is distributed under the terms of the Creative Commons Attribution License which permits any use, distribution, and reproduction in any medium, provided the original author(s) and the source are credited.

\section{References}

[1] European Commission (2013) Report from the Commission to the European Parliament and the Council: Renewable Energy Progress Report. COM(2013)175 final, SWD (2013) 102 final. European Commission, Brussels 
[2] Demirok E, Sera D, Rodriguez P et al (2011) Enhanced local grid voltage support method for high penetration of distributed generators. In: Proceedings of the 37th annual conference on IEEE Industrial Electronics Society (IECON'11), Melbourne, 7-10 Nov 2011, pp 2481-2485

[3] Robinson D, Kotony G (2008) A self-managing brokerage model for quality assurance in service-oriented systems. In: Proceedings of the 11th IEEE High Assurance Systems Engineering Symposium (HASE'08), Nanjing, 3-5 Dec 2008, pp 424-433

[4] Choi SS, Tseng KJ, Vilathgamuwa DM et al (2008) Energy storage systems in distributed generation schemes. In: Proceedings of the IEEE Power and Energy Society General Meeting: conversion and delivery of electrical energy in the 21st century, Pittsburgh, 20-24 Jul 2008, 8 p

[5] You S (2010) Developing virtual power plant for optimized distributed energy resources operation and integration. Ph D Thesis, Technical University of Denmark, Lyngby

[6] Directorate-General for the Information Society and Media, European Commission (2009) ICT for a low carbon economy: Smart electricity distribution networks. Dictus Publishing, St Louis

[7] Constantin A, Lazar RD, Kjær SB (2012) Voltage control in low voltage networks by photovoltaic inverters-PVNET.dk. Danfoss Solar Inverters A/S, Gråsten

[8] VDE-AR-N 4105 (2011) Generators connected to the lowvoltage-distribution network-technical requirements for the connection to and parallel operation with low-voltage distribution networks

[9] Connecting the sun (2012) Solar photovoltaics on the road to large-scale grid integration. European Photovoltaic Industry Association, Brussels, Belgium

[10] Stetz T, Marten F, Braun M (2013) Improved low voltage gridintegration of photovoltaic systems in Germany. IEEE Trans Sustain Energ 4(2):534-542

[11] Jadeja K (2012) Major technical issues with increased PV penetration on the existing electrical grid. MS thesis, Murdoch University, Perth

[12] Carvalho PMS, Correia PF, Ferreira LAF (2008) Distributed reactive power generation control for voltage rise mitigation in distribution networks. IEEE Trans Power Syst 23(2):766-772

[13] Tonkoski R, Lopes LAC, EL-Fouly THM (2011) Coordinated active power curtailment of grid connected PV inverters for overvoltage prevention. IEEE Trans Sustain Energy 2(2):139-147

[14] Masters C (2002) Voltage rise: The big issue when connecting embedded generation to long $11 \mathrm{kV}$ overhead lines. Power Eng J 16(1):5-12

[15] Paracha ZJ, Doulai P (1998) Load management: Techniques and methods in electric power system. In: Proceedings of the international conference on energy management and power delivery (EMPD'98), vol 1, Singapore, 3-5 Mar 1998, pp 213-217

[16] Marra F, Yang GY, Fawzy YT et al (2013) Improvement of local voltage in feeders with photovoltaic using electric vehicles. IEEE Trans Power Syst 28(3):3515-3516

[17] Esslinger P, Witzmann R (2012) Evaluation of reactive power control concepts for PV inverters in low-voltage grids. In: Proceedings of the CIRED workshop on integration of renewables into the distribution grid, Lisbon, Portugal, 29-30 May, 2012, 4 $\mathrm{p}$

[18] Chen M, Allen T (2013) A novel integrated vacuum tapping interrupter. In: Proceedings of the 22nd international conference and exhibition on electricity distribution (CIRED'13), Stockholm, 10-13 Jun 2013, 4 p

[19] BDEW e V (2008) Technical guidelines: Generating plants connected to the medium-voltage network. BDEW e V, Berlin
[20] Markiewicz H, Klajn A (2004) Power quality application guide. Copper Development Association, Lonton

[21] IEC 61400-21:2008 (2008) Wind turbines-part 21: measurement and assessment of power quality characteristics of grid connected wind turbines

[22] Rev 21. FGW e V (2010) Technical guidelines for power generating units-part 3: determination of electrical characteristics of power generating units connected to MV, HV and EHV grids. Rev 21. FGW e V, Berlin

[23] Clark K, Miller NM, Walling R (2010) Modeling of GE solar photovoltaic plants for grid studies. General Electric International Inc., Schenectady, NY

[24] Caldon R, Turri R, Prandoni V et al (2004) Control issues in MV distribution systems with large-scale integration of distributed generation. In: Proceedings of the 2010 IREP symposium: bulk power system dynamics and control-VI (IREP'10), Cortina d'Ampezzo, 22-27 Aug 2004, pp 583-589

[25] Miguel Jumperaz Goni (2013) Test platform development for large scale solar integration. Technical University of Denmark, Lyngby, Denmark

[26] Danish electricity supply (2008) Statistical survey. Danish Energy Association, Frederiksberg

[27] TLX inverterserien (2013) Danfoss Solar Inverters A/S, Gråsten

[28] G4400 BLACKBOX fixed power quality analyzer. Elspec, Caesarea, Israel

Miguel JUAMPEREZ was born in Pamplona, Spain, in 1986. He received the B.E. degree in electrical engineering from the Public University of Navarre (UPNA), Pamplona, Spain, in 2010, and the M.Tech. degree in electrical engineering from the Lappeenranta University of Technology (LUT), Lappeenranta, Finland, in 2013. In 2012, he joined the Centre of Electric Power and Energy (CEE), Department of Electrical Engineering, Technical University of Denmark (DTU), as a Master Student, and in 2013 became a Researcher assistant. During that time, his research interests included power electronics, power systems, power integration, PV power, reactive power compensation, voltage regulation and power quality.

Guangya YANG received the BE and ME in 2002, 2005, respectively from Shandong University, and $\mathrm{PhD}$ in 2008 from the University of Queensland, all in the field of electric energy system. He came to Denmark in 2009 as Postdoc and afterwards Scientist with Technical University of Denmark. He is currently Associate Professor with the Department of Electrical Engineering, Technical University of Denmark. His research interests are in electric energy system operation and control, wide area monitoring and protection, energy storage, system identification and application of optimisation techniques in the electric energy systems.

Søren B/EKHØJ KJ/ER received the MSc and $\mathrm{PhD}$ degrees in electrical engineering from Institute of Energy Technology, Aalborg University, DENMARK, in 2000 and 2005, respectively. He is currently with Danfoss Solar Inverters, DENMARK, where he holds a position as Lead System Architect. He was with the Section of Power Electronics and Drives, Aalborg University, from 2000 to 2004, as Research and Laboratory Assistant. He also taught courses on photovoltaic systems for terrestrial and space applications (power system for the AAU student satellite: AAU CubeSat). His main interests are switching inverters for photovoltaic applications, including power quality, grid voltage control, fault ride through, MPPT, SmartGrid and design optimization. He has also been involved in standardization work within safety of inverters, ancillary services for inverters and methods for evaluating inverters total efficiency. 